\title{
LEPIDOBROCA DE FRUTOS DO PEQUIZEIRO: UMA NOVA ESPÉCIE DO GÊNERO CARMENTA (LEPIDOPTERA: SESIIDAE)
}

Gilmar Poncio de Oliveira ${ }^{1}$

Marcelo Tavares de Castro ${ }^{2}$

\section{INTRODUÇÃO}

O pequizeiro (Caryocar brasiliense Camb.) é uma árvore bastante apreciada pela população do Cerrado brasileiro. Pertencente à família Caryocaraceae (Oliveira et al., 2008), possui crescimento frondoso, com porte entre 8 a $12 \mathrm{~m}$ de altura, dependendo do ambiente que está alocado no bioma Cerrado (Oliveira et al., 2008). Tem ocorrência desde o sul do Estado do Pará até o centro norte do Paraná, do Mato Grosso até São Paulo, mais comumente na área central do Brasil, incluindo Minas Gerais e alguns estados do Nordeste brasileiro (Almeida et al., 1998). Seus frutos constituem fonte de alimentação e renda para os moradores do Cerrado brasileiro, principalmente entre os meses de outubro a março, período em que os frutos amadurecem e caem, movimentando cerca de $50 \%$ da população local (Alencar et al., 2000) e é utilizado no preparo de pratos típicos, bem como óleos e bebidas (BIANCO \& PITELLI, 1986; OLIVEIRA ET AL., 2008; SIQUEIRA ET AL., 2012).

No começo da estação com maior densidade pluviométrica começa a emissão de botões florais (Vilela et al., 2008), essas flores irão gerar frutos com mesocarpo de coloração amarelo claro, epicarpo carnoso e endocarpo espinhoso (Almeida et al., 1998). 0 tempo de germinação desse fruto pode chegar a mais de um ano, sendo entre $5 \%$ e $60 \%$ sua taxa de germinação (Rocha \& Fernandes, 2009). O pequizeiro tem queda de folhas entre maio a agosto

1 Graduando da Faculdade ICESP/Promove de Brasília, Distrito Federal, Brasil. gilmarponcio@yahoo.com.br

2 Professor da Faculdade ICESP/Promove de Brasília, Distrito Federal, Brasil. marceloengflorestal@gmail.com 
(Magalhães, 2013), época mais seca do ano. Vilela et al. (2008) apresentam que o desfolhamento se dá no começo da estação seca.

Vários insetos já foram relatados em C. brasiliense. Representantes das ordens Lepidoptera, Coleoptera, Hemiptera, Hymenoptera, Diptera, Neuroptera, Orthoptera, Thysanoptera, entre outros, já foram encontrados associados ao pequizeiro em diferentes partes vegetais, incluindo ramos, folhas, caule e frutos (Barradas, 1972; Garcia, 1995; Lopes et al., 2003; Fernandes et al., 2004; Leite et al., 2006; Ferreira et al., 2009), muitos deles sendo possíveis visitantes, não causando danos aparentes.

Uma dos insetos de maior importância para o pequizeiro causa a queda prematura de frutos. Esses são atacados por lagartas de Carmenta sp. (Lepidoptera: Sesiidae), na qual elas destroem o interior comestível do fruto, tornando seu consumo inviável, com perdas de $50 \%$ da produção, em média (LOPES ET AL., 2003; CARVALHO \& CASTRO, 2016).

A espécie de Carmenta encontrada nos pequizeiros tem semelhança com a C. foraseminis (Sesiidae), que ataca o cacaueiro (Theobroma cacao L.) (PUCHI, 2005). Porém, poucos estudos a respeito do inseto foram realizados. Este trabalho tem como objetivo estudar a incidência de Carmenta sp. durante os meses de produção de frutos de pequi e fornecer maiores detalhes sobre a biologia, ecologia e morfologia do inseto.

\section{DELINEAMENTO EXPERIMENTAL}

Foram vistoriados 50 pequizeiros no Parque Nacional de Brasília (PNB), Distrito Federal (coordenadas: $15^{\circ} 44^{\prime} 07.6^{\prime \prime} \mathrm{S} ; 47^{\circ} 56^{\prime} 02.7^{\prime \prime} \mathrm{W}$ ), 20 árvores em regiões distintas da Asa Norte, Distrito Federal (coordenadas $15^{\circ} 44^{\prime} 38.2^{\prime \prime} \mathrm{S} ; 47^{\circ} 53^{\prime} 04.4^{\prime \prime} \mathrm{W}$ ) e 30 árvores no Parque Ecológico do Superior Tribunal de Justiça, Distrito Federal (coordenadas $15^{\circ} 48^{\prime} 37.6^{\prime \prime} \mathrm{S} ; 47^{\circ} 51^{\prime} 54.4^{\prime \prime} \mathrm{W}$ ), e arredores, com pequizeiros situados em área de Cerrado sensu stricto, durante os meses de setembro de 2016 a janeiro de 2017 (Figura 1). Durante as coletas, foram observadas a presença de lagartas nos troncos, ramos, folhas e frutos. Os frutos coletados foram separados em secos (provavelmente com queda em períodos anteriores ao da coleta) e verdes recém-caídos, de acordo com o mês da coleta. 


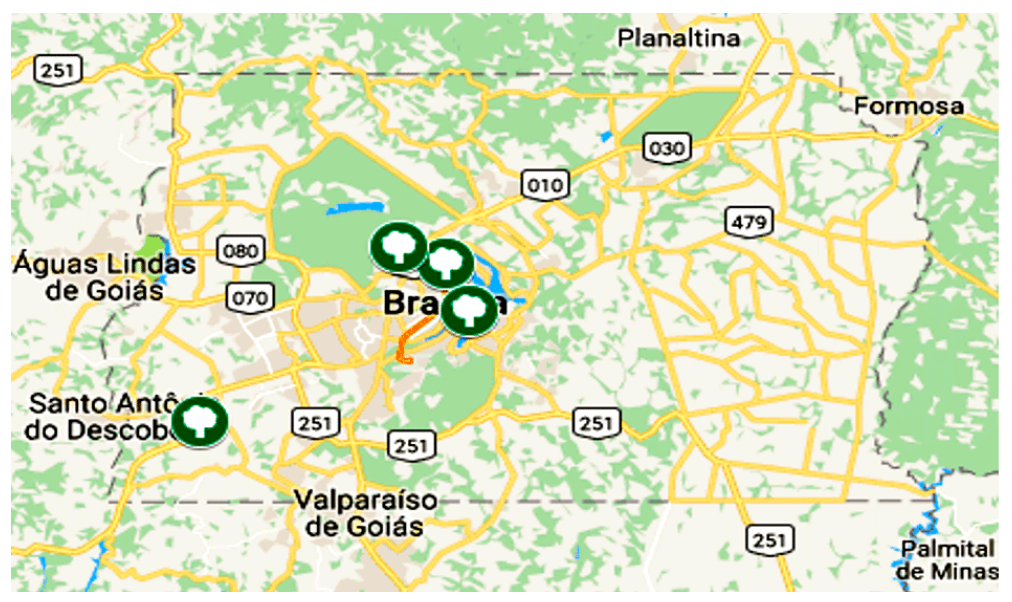

Figura 1: Áreas vistoriadas no Distrito Federal, Brasil, contendo pequizeiros e utilizadas nesse estudo. Fonte: Google Maps.

Foi realizada a contagem de frutos atacados, número de lagartas encontradas e número total de adultos emergidos. Além disso, observações acerca da espécie foram feitas no campo, como presença de excrementos, orifícios e outros sinais que indicassem a presença do inseto no interior dos frutos. Alguns frutos foram parcialmente abertos para verificar a presença ou ausência de lagarta/ pupa no seu interior. Foi considerado fruto atacado aqueles que continham orifícios e excrementos típicos do ataque de Carmenta sp, conforme Lopes et al. (2003) e CARVALHO \& CASTRO (2016).

Alguns dos frutos coletados foram transportados em sacos plásticos rotulados para observação em laboratório. No laboratório, foram colocadas em potes plásticos modificados para a anotação das datas de emergência dos adultos. Nesse local, os frutos foram vistoriados diariamente, observando sua evolução desde a lagarta até sua morte quando adulto. Logo após a sua morte, algumas mariposas foram alfinetadas, montadas e secas e outras foram colocadas em potes plásticos contendo álcool 70\% para preservação. Para a descrição morfológica do inseto, foram utilizados vinte e cinco lagartas e vinte e cinco adultos. Para a confirmação do gênero e contribuições acerca da espécie, foi utilizada chave específica para a família Sesiidae e comparações com espécies já descritas na literatura foram realizadas (PUCHI, 2005). 


\section{RESULTADOS E DISCUSSÃO}

Durante todo o experimento, foram coletados cerca de 500 frutos e obtidos 73 adultos de Carmenta sp, durante a criação em laboratório. Nas coletas em campo, não foram encontradas mariposas no local, apenas imaturos. Ao todo 28 lagartas foram coletadas separadas para estudos morfológicos, sendo que destas, 25 foram utilizadas. Portanto, 101 indivíduos de Carmenta sp. foram capturados durante todo o estudo. De todos os frutos secos coletados, $20(13,51 \%)$ apresentaram sinais de ataque do inseto. Com relação aos frutos verdes recém-caídos, 200 (60,60\%) estavam predados, corroborando Lopes et al. (2003) e Carvalho \& Castro (2016). Não foram observadas lagartas em outras partes da planta, permanecendo, assim, um mistério quanto ao seu hábito alimentar na ausência de frutos de pequi.

A presença de orifícios de abertura feitos pela lagarta nos frutos consiste em um dos principais sinais do ataque do inseto, na qual pode conter excrementos e teia. As lagartas, após construírem o orifício, vão em direção às amêndoas, danificando o mesocarpo e o endocarpo dos frutos, e uma grande presença de dejetos é encontrada em seu interior (Figura 2-A e B). Os orifícios são utilizados primordialmente para entrada do inseto no interior do fruto e posteriormente para a saída do adulto, de forma que a pupa é projetada para fora, fato observado também por LOPES ET AL. (2003).
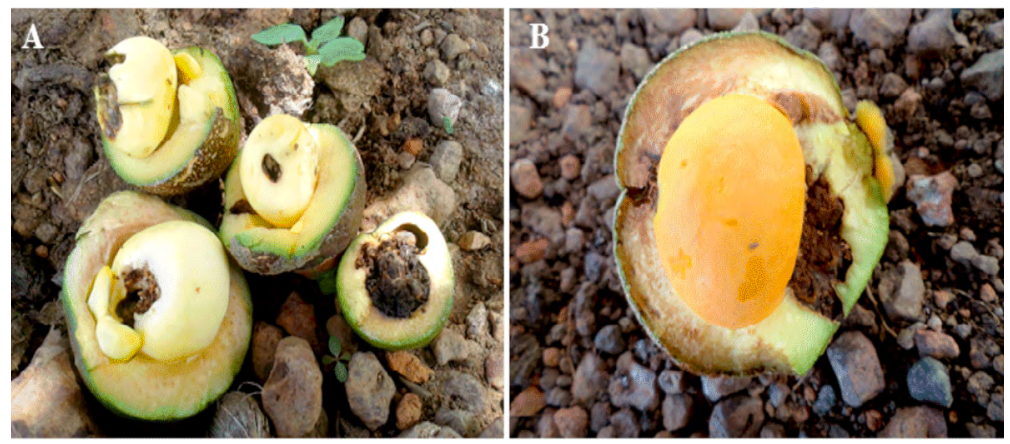

Figuras 2.A e 2.B: A) Ataque de Carmenta sp. em frutos de pequi; B) Detalhe dos frutos danificados pela lagarta e excrementos do inseto

Fonte: Construção dos autores. 2017. 
É importante observar que os frutos predados não necessariamente continham lagartas. Portanto, mesmo que um fruto apresentasse sinais de ataque da lagarta no exterior, ou seja, presença de excrementos ou orifícios causados pela lagarta, interiormente poderia não ter o inseto, o que sugere que a Carmenta pode danificar externamente e internamente o fruto e não necessariamente completar o seu ciclo dentro dele. Os danos provocados pelas lagartas nos frutos já foram bem descritos e relatados por Lopes et al. (2003) e Carvalho \& Castro (2016), onde os autores relatam perdas de $50 \%$ em média dos frutos, corroborando os dados aqui obtidos.

Com relação ao ataque de frutos nos diferentes meses de coleta, o mês de novembro foi o que mais apresentou frutos secos atacados e no mês de dezembro e janeiro mais frutos verdes atacados (Figura 3). Portanto, os meses de maior incidência de ataque compreende entre os meses de novembro e janeiro, período mais propício para tomar medidas preventivas e curativas de controle da praga. Resultados semelhantes foram obtidos por Benassi et al. (2013), onde $C$. foraseminis foi reportada em frutos de cacau no período de novembro a janeiro, época propícia ao ataque do inseto.

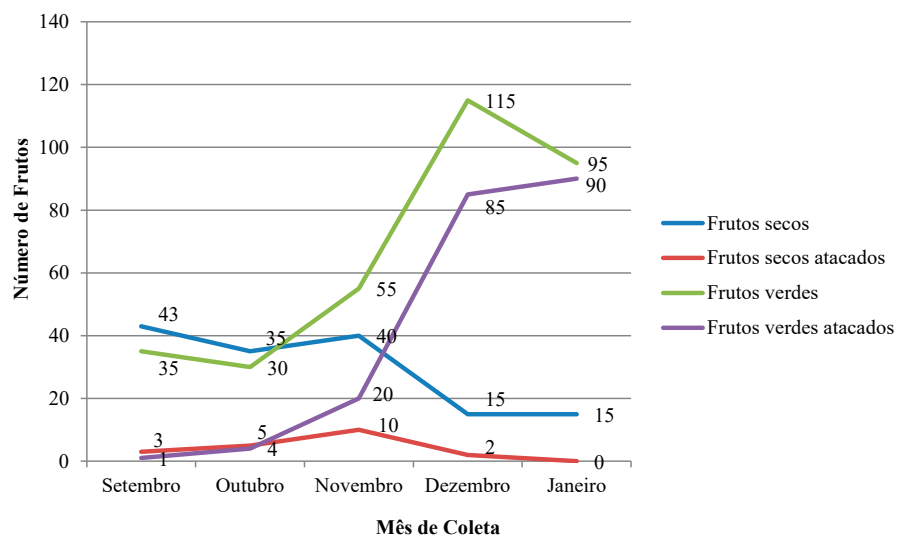

Figura 3. Incidência de Carmenta sp. em frutos secos e verdes de acordo com os meses coletados no Distrito Federal

As larvas encontradas no interior dos frutos de pequi são de cor branca a amarelo claro e a cabeça mais estreita que o pronoto. Possuem em média $16 \mathrm{~mm}$ de comprimento, muito vorazes ao 
atacar os frutos e com hábitos crípticos, ou seja, altamente fotofóbicas, fatos também observados por PUCHI (2005). Os adultos de Carmenta sp. (Figura 4) que atacaram o pequi se assemelharam morfologicamente e biologicamente com a Carmenta foraseminis (Eichlin, 1995), que ataca frutos do cacaueiro (Theobroma cacao L.) (EICHLIN, 1995; PUCCHI, 2005; BENASSI ET AL., 2013; CASTAÑEDA ET AL., 2015). As mariposas analisadas possuíram corpo negro, com bandas dorsais amarelas, delimitando os segmentos abdominais. Duckworth \& Eichlin (1974) afirmam que os membros da família Sesiidae apresentam grande variação morfológica e que o estudo da genitália masculina e feminina é preponderante na correta identificação da espécie. Além de $C$. foraseminis, a espécie Carmenta theobromae (Busck, 1910) também já foi relatada atacando a espécie (Eichlin, 1995; Castañeda et al., 2015), porém os danos nos frutos são diferentes, onde raramente $C$. theobromae consome as sementes. Segundo Puchi (2005), mais de vinte espécies de Carmenta já foram relatadas no Brasil, porém pouco se sabe a respeito de suas hospedeiras.

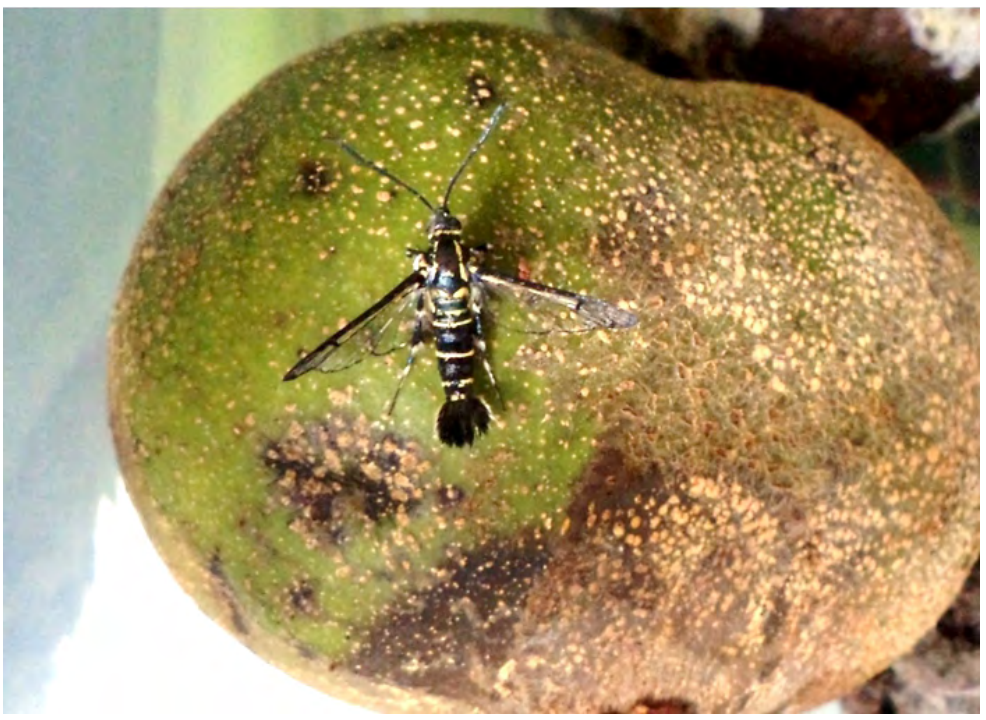

Figura 4. Mariposa de Carmenta sp. sobre fruto de pequi

Segundo descrições de Jiménez \& Cabaña (2006), os ovos de $C$. foraseminis são ovais e medem aproximadamente 0,6 por $0,3 \mathrm{~mm}$, 
as larvas são esbranquiçadas, sua cabeça tem coloração marrom. As lagartas penetram nos frutos de cacau e posteriormente alimentam-se das sementes. O inseto possui pupa na cor marrom, seus adultos tem cor escura, com asas quase transparentes, apresentam escamas escuras e tem faixas amarelas no seu dorso e ventre. Sua lagarta produz um excremento semelhante a borra de café, após penetrarem a poupa do cacau, o que se assemelha ao verificado nas frutas do pequizeiro. Portanto, suspeita-se que a espécie em estudo se trata de $C$. foraseminis.

Alguns autores relataram que as espécies de Carmenta são hospedeiras específicas, ou seja, se alimentam apenas de uma ou algumas hospedeiras, podendo, assim, passíveis de serem utilizadas em programas de controle biológico de plantas invasoras (FORNO ET AL., 1991; CORDO ET AL., 1995; OSTERMEYER AND GRACE, 2007; DHILEEPAN ET AL., 2012. Além disso, segundo Hairms \& Aiello (1995), C. foraseminis é a única espécie dentro do gênero que se alimenta de sementes, configurando em um hábito distinto das demais.

\section{CONSIDERAÇÕES FINAIS}

Há suspeitas de que a espécie que ataca frutos de pequizeiros seja a $C$. foraseminis, através de semelhanças morfológicas e também pelo seu hábito alimentar. Porém, estudos mais detalhados acerca da morfologia do inseto devem ser realizados para tal confirmação. Além disso, com este estudo foi possível observar que o período que maior ataque de frutos de pequi pela Carmenta sp. no Distrito Federal compreende os meses de novembro a janeiro, onde há maior queda de frutos verdes.

\section{REFERÊNCIAS}

ALMEIDA, S. P.; PROENCA, C. E. B.; SANO, S. M.; RIBEIRO, J. F. Cerrado: espécies vegetais uteis. Planaltina: EMBRAPA-CPAC, 1998.

ALENCAR, G. 2000. Pequizeiros enfrentam riscos de extinção. Hoje em Dia, Belo Horizonte, 13 de fev., p. 07.

BARRADAS, M. M. 1972. Informações sobre a floração, frutificação e dispersão pequi Caryocar brasiliense Camb. (Caryocaraceae) Ciência e Cultura, v. 24, p.1063- 1068. 
BENASSI, V. L. C., SOUZA, C. A., VALENTE, F. I., LENZI, J. C. 2013. Carmenta foraseminis (Lepidoptera: Sesiidae), nova broca de frutos de cacau no Brasil. Revista de Agricultura, Piracicaba, SP, v. 88, p. 70-75.

BIANCO, S.; PITELLI, R.A. 1986. Fenologia de quatro espécies de frutíferas nativas dos cerrados de Selvíria, MS. Revista Pesq. Agropec. Bras., v.21, n.11, p.1229-1232.

BUSCK, A. 1910. List of Trinidad microlepidoptera with descriptions of new species. Bulletin of the U.S. Department of Agriculture. Trinidad, v. 9, p. 241-245.

CARVALHO, R. L. S. \& CASTRO, M. T. 2016. Ataque de Carmenta sp. (Lepidoptera: Sesiidae) em pequizeiros no Distrito Federal, Brasil. Revista Científica Eletrônica de Engenharia Florestal, v. 28, n. 1, p. 14-22.

CASTAÑEDA, Y. V.; GUTIÉRREZ, J. M.; RUIZ, S. B. M.; HERNÁNDEZ-BAZ, F. 2015. Ocurrencia de los barrenadores Carmenta foraseminis Eichlin y Carmenta theobromae (Busck) (Lepidoptera: Sesiidae) en Theobroma cacao L., em el Deparamento de Antioquia-Colombia. Boletín del Museo de Entomología de la Universidad del Valle, v. 16, n. 1, p. 34-38.

CORDO, H. A.; DELOACH, C. J.; FERRER, R. 1995. Host range of the Argentine root borer Carmenta haematica (Ureta) (Lepidoptera: Sesiidae), a potential biocontrol agent for snakeweeds (Gutierrezia spp.) in the United States. Biological Control, v. 5, p. 1-10.

DHIELLEPAN, K.; TREVINO, M.; VITELLI, M. P.; WILMOT SENARATNE, K. A. D.; McCLAY, A. S.; McFADYEN, R. E. 2012. Introduction, Establishment, and Potential Geographic Range of Carmenta sp. nr ithacae (Lepidoptera: Sesiidae), a Biological Control Agent for Parthenium hysterophorus (Asteraceae) in Australia. Environmental Entomology, v. 41, n. 2, p. 317-325.

DUCKWORTH, W. \& EICHLIN, T. D. 1974. Clearwing moths of Australia and New Zealand (Lepidoptera: Sesiidae). Smithsonian Contr Zool. 180 p.

EICHLIN, T. D. 1995. A new Panamaninan clearwing moth. Journal of Lepidopterists Society, v. 49, p. 39-42.

FERNANDES, L. C.; FAGUNDES, M.; SANTOS, F. A.; SILVA, G. M. 2004. Abundância de insetos herbívoros associados ao pequizeiro (Caryocar brasiliense Cambess.). Revista Árvore, v. 28, p. 919-924.

FERREIRA, G. A.; ROCHA SANTOS VELOSO, V.; VELOSO NAVES, R.; NASCIMENTO, J. L.; CHAVES, L. J. 2009. Biodiversidade de insetos em Pequizeiro (Caryocar brasiliense, Camb.) no cerrado do Estado de Goiás, Brasil. Agrociência, v. 13, n. 2, p. 14-31.

FORNO, I. W.; KASSULKE, R. C.; DAY, M. D. 1991. Life cycle and host testing procedures for Carmenta mimosa Eichlin and Passoa (Lepidoptera: Sesiidae), a biological control agent for Mimosa pigra L. (Mimosaceae) in Australia. Biological Control, v.1, p. 309-315.

GARCIA, A. H. 1995. Ação do parasitóide Anatatus sp. (Eupelmidae- Hymenoptera) em ovos de Dirphia rosacordis Walker, 1855 (Lepidoptera- Saturnidae) em pequi- 
zeiro (Caryocar brasiliense Cambess). Anais... Escola de Agronomia e Veterinária da UFG, v. 25, p. 161-164.

HARMS, K. E.; AIELLO, A. 1995. Seed-boring by tropical clearwing moths (Sesiidae): aberrant behavior or widespread habit? Journal of the Lepidopterists Society, v. 49, n.1, p. $43-48$.

JIMÉNEZ, R. V. N.; CABAÑA, W. 2006. Control de insectos perforadores de la mazorca del cacao en la zona central de Venezuela. INIA Divulga, n.7, p.19-26.

LEITE, G. L. D.; VELOSO, R. V. S.; REDOAN, A. C.; LOPES, P. S. N. E MACHADO, M. M. L. 2006. Artropodes (Arthropoda) associados a mudas de pequizeiro Caryocar brasiliense Cambes. (Caryocaraceae). São Paulo: Arquivo Instituto Biológico, v. 73, p. 365-370.

LOPES, P. S. N., Souza, J. C., Reis, P. R., Oliveira, J. M., Rocha, I. D. F. 2003. Caracterização do ataque da broca dos frutos de pequizeiro. Revista Brasileira de Fruticultura, Jaboticabal - SP, v. 25, n. 3, p. 540-543.

MAGALHÃES, M. R. Padrões espaciais e temporais da fenologia de três espécies arbóreas na região do Alto Jequitinhonha. 2013. 86p. Dissertação (Mestrado em Ciência Florestal), Universidade Federal dos Vales do Jequitinhonha e Mucuri.

OLIVEIRA, M. E. B.; GUERRA, N. B.; BARROS, L. M.; ALVES, R. E. 2008. Aspectos Agronômicos e de Qualidade do Pequi. Embrapa Agroindústria Tropical, Fortaleza,

OLIVEIRA, T. F.; AGUIAR, L. M. S.; CAMARGO, N.F. Visitantes florais e potenciais polinizadores secundários de Caryocar brasiliense Camb. In: II SIMPÓSIO INTERNACIONAL SAVANAS TROPICAIS, 2008, Brasília, DF. Anais... Brasília: SIST, 2008.

OSTERMEYER, N. \& GRACE, B. S. 2007. Establishment, distribution, and abundance of Mimosa pigra biological control agents in northern Australia: implications for biological control. BioControl, v. 52, p. 703-720.

PUCHI, N. D. 2005. Caracterización morfológica de los Sesiidae (Insecta: Lepidoptera) perforadores del fruto del cacao (Theobroma cacao L.), presentes en la región costera del estado Aragua, Venezuela. Entomotropica, v.20, n.2, p. 97-111.

ROCHA, J. P., FERNANDES, J. S. C. Fatores ambientais e genéticos na germinação desementes de pequizeiro (Caryocar brasiliense Camb.). In: V CONGRESSO BRASILEIRODE MELHORAMENTO DE PLANTAS, 2009, Guarapari, ES. Anais... Guarapari: SBMP,2009.

SIQUEIRA, B. S.; ALVES, L. D.; VASCONCELOS, P. N.; DAMIANI, C.; SOARES JÚNIOR, M. S. 2012. Pectina extraída de casca de pequi e aplicação em geléia light de manga. Revista Brasileira de Fruticultura, v.34, n.2 p.560-567.

VILELA, G. F.; CARVALHO, D.; VIEIRA, F. A. Fenologia de Caryocar brasilense Camb (Caryocaraceae) no alto Rio Grande, sul de Minas Gerais. Revista Cerne, Lavras, v. 14, n. 4, p. 317-329, out.-dez., 2008. 